\title{
X. THE SOLUBILITY OF GLYCOGEN.
}

\author{
By MARGARET KERLY. \\ From the Department of Physiology and Biochemistry, \\ University College, London.
}

(Received December 27th, 1929.)

IN the course of an investigation on glycogen, the solubility of a sample prepared in the usual way by boiling tissue with $60 \% \mathrm{KOH}$ was compared with that of a sample prepared from an aqueous extract of tissue dehydrated by alcohol, and it was observed that the former sample reached a stable value very much more rapidly than the latter. It was therefore decided to prepare glycogen from several sources, without boiling with potassium hydroxide and to determine the solubilities of the products obtained. In the course of the work it was found necessary to revise the method employed for estimating glycogen, and, in order to do this, the solubility of glycogen in aqueous alcohol was investigated.

\section{Method of preparation.}

Since the usual method of preparing glycogen by boiling tissue with $60 \%$ potassium hydroxide appeared to affect the solubility of the product, it was considered advisable to use a method less likely to have a chemical action on the carbohydrate complex. Dehydration of the tissue by alcohol and subsequent extraction with water was the method selected, except in one case (frog muscle) where the tissue was extracted with $4 \%$ trichloroacetic acid, and the glycogen precipitated with alcohol. Three sources of glycogen were used: mussel (whole body), frog (skeletal muscle) and rabbit (liver). The method employed varied slightly in each case.

Mussel (Mytilus edulis). Mussels were killed; and the tissue was dehydrated, by being put into alcohol. For the first batch boiling $80 \%$ alcohol was used, for the second, cold $80 \%$ alcohol. The solid residue was filtered off, dried with absolute alcohol and with ether, exposed to the air to remove all traces of ether, and finally ground in a mortar. Glycogen was extracted from this powder with water: the proteins, denatured by the treatment with alcohol, were soluble only to a small extent.

To test the completeness of extraction, a portion of the mussel powder was extracted 18 times with small quantities of water at $0^{\circ}$, and the glycogen in each extract and in the residue estimated separately (Fig. 1, curve $a$ ). The experiment was repeated, using water at $25^{\circ}$ (curve $b$ ), but the amounts extracted were not appreciably greater. Grinding with sand between each extraction gave much more satisfactory results (curve c), and it was concluded that the difficulty of extraction was mechanical. From Fig. 1 it is apparent that the seventh and subsequent extracts contain negligible amounts of glycogen; in later work, therefore, the mussel powder was extracted six times. 
Glycogen values are given throughout this paper in terms of the reducing sugar found after 3 hours' hydrolysis by $2.2 \%$ by wt. hydrochloric acid. It is usual to multiply this by 0.927 to convert to glycogen. This assumes a $97 \%$ hydrolysis, but working with a purified sample of glycogen prepared by Dr Slater, it was never found possible to reach so high a value, the mean obtained being $94 \%$, corresponding to a conversion factor 0.957 .

The glycogen in the water extract was purified by precipitation with two volumes of alcohol.

The nitrogen and phosphorus contents of successive precipitates were examined, and it was found that the ratio of the nitrogen and phosphorus to glycogen fell considerably after the first precipitation, showed no decided change after the second, and after the third precipitation the nitrogen values rose, phosphorus remaining nearly steady (Table I). This unexpected result was repeated in each preparation examined, and suggests that the glycogen molecule in the natural state may contain nitrogen, and possibly also phosphorus. For the third precipitation it was necessary to add an electrolyte to coagulate the glycogen; a few drops of a normal solution of potassium acetate were used.

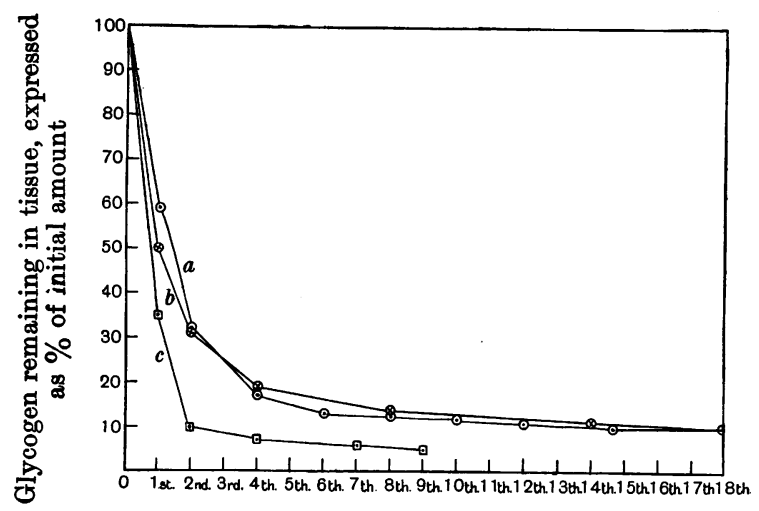

Fig. 1. Extraction of glycogen from dried mussel powder.

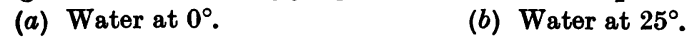

(c) Water + grinding with sand at $15^{\circ}$.

Table I.

Ratio mg. phosphorus and $\mathrm{mg}$. nitrogen to $100 \mathrm{mg}$. glycogen.

\begin{tabular}{|c|c|c|c|c|c|c|c|c|}
\hline & \multicolumn{2}{|c|}{$\begin{array}{c}\text { Untreated } \\
\text { extract }\end{array}$} & \multicolumn{2}{|c|}{ After 1 ppt. } & \multicolumn{2}{|c|}{ After 2 ppt. } & \multicolumn{2}{|c|}{ After 3 ppt. } \\
\hline & $\overbrace{\mathrm{P}}$ & $\widehat{N}$ & $\overparen{P}$ & $\vec{N}$ & $\overparen{P}$ & $\vec{N}$ & P & $\vec{N}$ \\
\hline$\underset{\text { Prep. }}{\text { A }}$ & $\begin{array}{l}0.91 \\
0.85\end{array}$ & $\begin{array}{l}1.91 \\
1.74\end{array}$ & $\begin{array}{l}0.20 \\
0.75\end{array}$ & $\begin{array}{l}0.55 \\
0.57\end{array}$ & $\begin{array}{l}0.21 \\
0.63\end{array}$ & $\begin{array}{l}0.56 \\
0.54\end{array}$ & $\begin{array}{l}0.17 \\
0.64\end{array}$ & $\begin{array}{l}0.98 \\
0 \cdot 70\end{array}$ \\
\hline
\end{tabular}

In order to remove small amounts of protein that might still be associated with it, the glycogen was dissolved in $4 \%$ trichloroacetic acid, filtered and dialysed.

After three precipitations with alcohol, dialysis of the product produced a rise in the ratio of nitrogen to glycogen. If the glycogen were first dissolved in $4 \%$ trichloroacetic acid, the small amount of insoluble matter filtered off, and the solution dialysed, there was a larger rise. If, instead of being dissolved in trichloroacetic acid, the glycogen were boiled for 2 hours with $60 \%$ potash, the ratio of nitrogen to glycogen fell very considerably (Table II). 


\author{
Table II.
}

Ratio mg. nitrogen to $100 \mathrm{mg}$. glycogen.

\begin{tabular}{cccc} 
& \multicolumn{4}{c}{ After dissolving in $4 \%$} \\
Prep. C after 2 ppt. & After & trichloroacetic acid with & After boiling with \\
with $66 \%$ alcohol & dialysis & subsequent dialysis & $60 \%$ potash \\
0.77 & 0.81 & 0.83 & 0.30
\end{tabular}

After dialysis the glycogen was precipitated a third time in $66 \%$ alcohol and then dried in a vacuum desiccator over calcium chloride. This should give the half-hydrate, $\mathrm{C}_{6} \mathrm{H}_{10} \mathrm{O}_{5}, \frac{1}{2} \mathrm{H}_{2} \mathrm{O}$ [Slater, 1924].

Rabbit. The rabbit liver was removed immediately the animal was killed and was minced into ice-cold $94 \%$ alcohol, filtered and the residue reextracted with ice-cold alcohol, filtered and the residue dried in air and ground in a mortar. Glycogen was extracted from this powder in the same manner as for mussels.

Frog. The frogs' muscles were ground in $4 \%$ trichloroacetic acid and the extract treated as the water extract from mussel powder, except that the glycogen was not again dissolved in trichloroacetic acid.

\title{
Method of determining solubility.
}

Preliminary experiments showed that glycogen did not go into solution completely for several days. This made it necessary to ensure that the material should remain sterile during the time of the experiment. Small two-necked flasks were used, one neck closed with a rubber cap, the other stoppered with cotton wool and covered with a glass cap. A small amount of glycogen (1 to $0 \cdot 3 \mathrm{~g}$.) was placed in the flask and sterilised for 30 minutes in steam on 3 consecutive days; an amount of sterile water, insufficient to dissolve all this glycogen, was injected through the rubber cap, and the flask was placed in a thermostat. At intervals of 6 to 60 hours small amounts (about $0.5 \mathrm{cc}$.), were withdrawn by means of a hypodermic syringe, placed in a centrifuge cup, covered with a drop of liquid paraffin to prevent evaporation, and centrifuged for 20 minutes. $0.1 \mathrm{cc}$. of the solution was used for the estimation of the glycogen. That the solutions remained sterile was shown by plating out a drop on agar at the conclusion of the experiment. (Previous experiments with unsaturated glycogen solutions showed that the strength of the solution was not altered by centrifuging in these conditions.)

\section{Method of estimating glycogen.}

Since the quantities of glycogen available were small it was necessary to use a micro-method for estimation. The glycogen was still associated with appreciable quantities of nitrogen and phosphorus, and was therefore possibly contaminated by reducing substances, so it was considered desirable to use the full method of estimation by boiling with $60 \%$ potassium hydroxide and not merely to hydrolyse the solution with acid. 
With the exception of the method of de Jongh and Planelles [1924], depending on the turbidity of glycogen solutions precipitated by alcohol in the presence of ether, which is not suitable for accurate estimations, all micro-methods for the estimation of glycogen are modifications of Pflüger's method [1904], and depend on the destruction of lower carbohydrates by boiling with $60 \%$ potassium hydroxide, and precipitation of glycogen from the resulting solution with alcohol. Evans [1925] has pointed out that when estimating small quantities of glycogen in a considerable mass of tissue, involving the use of relatively large volumes of solution, there is a loss of glycogen, increasing with increasing volume of fluid, pointing strongly to the solubility of glycogen in aqueous alcohol, at least in the presence of a high concentration of potash. Holmes and Holmes [1926] carried out some experiments to test the recovery of glycogen added to a potassium hydroxide-alcohol mixture of the concentration used in their estimations, and obtained on the average a recovery of over $100 \%$, and concluded that there is no appreciable loss through solubility of glycogen in alcohol. Possibly they obtained such good values because they added solid glycogen, instead of precipitating from a solution, and, as the results of the solubility determinations show, glycogen may be very slow in dissolving. In a modification of the Pflüger method introduced by Bierry and Gouzon [1928], glycogen is not precipitated after alkaline hydrolysis, but acid is added and an acid hydrolysis carried out on the same solution. Proteins are afterwards removed with mercurio nitrate, and the sugar is estimated. This procedure avoids loss of glycogen by solution in alcohol, but might cause an additive error, since any reducing substance not destroyed in either the alkaline or the acid hydrolysis, and not precipitated by mercuric nitrate, would be estimated as sugar.

To find whether any glycogen remained in solution after precipitation with alcohol, when using a micro-modification of the Pflüger method, the residual alcoholic solution from a glycogen estimation (the tissue used in this, and in all other estimations when working out the method for estimating glycogen, was, for convenience, skeletal muscle from the frog) was evaporated nearly to dryness, washed with $1 \mathrm{cc}$. of absolute alcohol and centrifuged, and the glycogen estimated in the residue by acid hydrolysis and determination of the reducing power.

\section{Table III.}

Apparent solubility of glycogen from frog muscle in $\mathbf{7 5} \%$ alcohol.

$0 \cdot 2-0.3 \mathrm{~g}$. muscle (or in last 3 experiments $0 \cdot 1$ cc. glycogen solution), were boiled with 2 cc. $60 \%$ potassium hydroxide for $2 \frac{1}{2}$ hours, neutralised with glacial acetic acid, filtered and 3 volumes absolute alcohol added, making $20 \mathrm{cc}$. in all.

mg. glycogen ppt.
by alcohol
0.59
0.88
0.59
$1 \cdot 10$
1.09
0.065
0.054
-5.159
9.50
9.50
6.83

mg. glycogen recovered
from alcohol
$0 \cdot 166$
$0 \cdot 128$
$0 \cdot 166$
$0 \cdot 227$
$0 \cdot 189$
$0 \cdot 091$
$0 \cdot 295$
$0 \cdot 241$
$0 \cdot 172$
$0 \cdot 143$
$0 \cdot 156$
$0 \cdot 197$

Apparent solu
glycogen in 75
mg. per 100
0.83
0.64
0.83
1.14
0.95
0.46
1.48
1.21
0.86
0.72
0.78
0.99

The results (Table III), show clearly the presence of some reducing substance, probably, at least in part, glycogen. A reducing substance stable to alkaline hydrolysis, soluble in $75 \%$ alcohol, and relatively insoluble in absolute alcohol would be included in this estimation. If there should be 
such a substance present in the muscle in variable quantity, it would account for the irregularity of the results.

Methyl alcohol and acetone were also tried as precipitants for glycogen, but failed to give as good results as ethyl alcohol.

As these results indicated that glycogen had an appreciable solubility in $75 \%$ alcohol, but did not give accurate figures, it was decided to study the problem of the solubility of glycogen in aqueous alcohol using purified glycogen and varying strengths of alcohol, so that the influence of other substances present in muscle should be eliminated.

\section{Solubility of glycogen in aqueous alcohol.}

In practice it was found best to estimate the solubility by an indirect method. A known quantity of glycogen in aqueous solution was treated with alcohol in amounts calculated to give 50,60, 66, 75, 80 and $90 \%$ alcohol by volume. The glycogen precipitated by this treatment was separated by centrifuging and estimated. The solubility of glycogen in the aqueous alcohol was calculated from the difference between the amount of glycogen precipitated and the amount originally taken. On account of the slight solubility of glycogen, very small quantities must be used in order to show a difference that can be measured with any degree of accuracy. The glycogen used was a highly purified sample from Mytilus, kindly supplied by Dr Slater. It is generally agreed that to precipitate pure glycogen satisfactorily from aqueous solution by the addition of alcohol is difficult. The turbid solution will not coagulate and settle with any regularity, even after centrifugalisation for 30 minutes. One drop of a $60 \%$ potassium chloride solution was therefore added, making a final concentration of $0.6 \%$ potassium chloride (Fig. 2, curve $a$ ).

When estimating glycogen after boiling with potassium hydroxide there is always a high concentration of salt or of alkali present, so the solubility was also determined in the presence of $21 \%$ potassium acetate, $42 \%$ potassium trichloroacetate, $15 \%$ potassium chloride, and $15 \%$ potassium hydroxide, these being approximately the concentrations reached when estimating glycogen. In each case the electrolyte was added to the glycogen solution before the alcohol, and subsequent procedure was that described above. The results are shown in Fig. 2. The solubility in the presence of $4 \%$ trichloroacetic acid was also determined, since this concentration of trichloroacetic acid is frequently used for extracting tissue.

In previous methods of estimating glycogen the concentration of alcohol used for precipitating glycogen has varied considerably. Pflüger [1904] used $57 \%$; Kahn [1922] in a micro-method 52.5 \%; Evans [1926] for plain muscle $66 \%$. From the curves in Fig. 2 it is clear that it is useless to employ higher .concentrations of alcohol than $66 \%$ when the original volume of solution is fixed, since the loss due to increased final volume more than counterbalances the gain from decreased solubility. When the final volume is the limiting factor 
it is definitely better to use $66 \%$ rather than $75 \%$, as it is possible to use a larger initial volume and the percentage loss is lessened.

Pflüger [1904] precipitated glycogen from the alkaline solution after diluting with two volumes of water; in some more recent methods the alkali is neutralised, usually with glacial acetic acid, in order to precipitate protein, and this is filtered off before alcohol is added. It is apparent from Fig. 2 that glacial acetic acid is not a suitable acid to use, as the solubility of glycogen is higher when potassium acetate is present than in the presence of either potassium trichloroacetate, potassium chloride or un-neutralised $\mathrm{KOH}$. It would seem that there is little to choose from the point of view of solubility alone between these last three conditions. It is probably better to neutralise, as this undoubtedly removes considerable quantities of protein. When hydro-

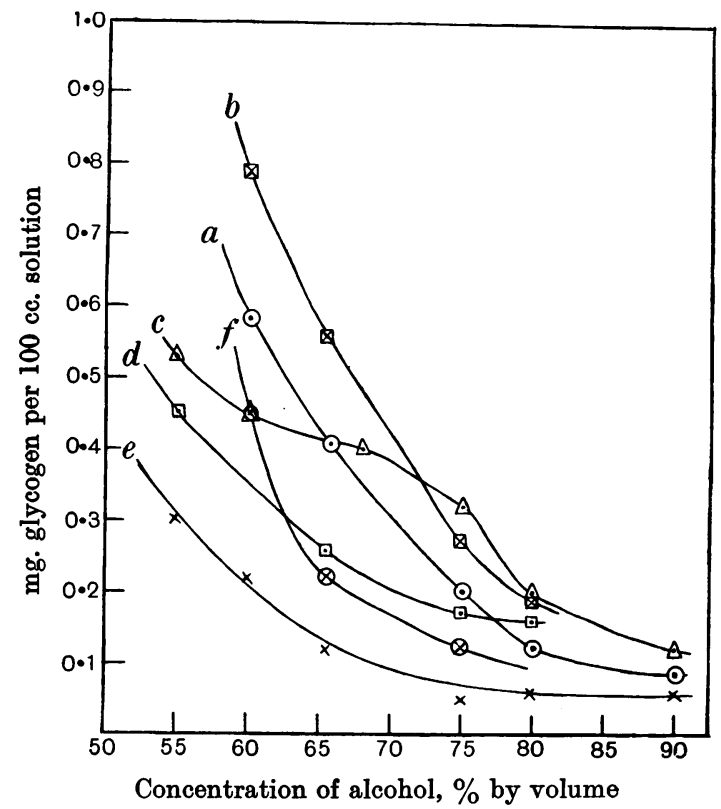

Fig. 2. The solubility of glycogen in aqueous alcohol.
(a) Alcohol $+0 \cdot 6 \% \mathrm{KCl}$.
(b) Alcohol $+4 \% \mathrm{CCl}_{3} \mathrm{COOH}$.
(c) Alcohol $+21 \% \mathrm{CH}_{3} \mathrm{COOK}$.
(d) Alcohol $+42 \% \mathrm{CCl}_{3} \mathrm{COOK}$.
(e) Alcohol $+15 \% \mathrm{KCl}$.
(f) Alcohol $+15 \% \mathrm{KOH}$.

chloric acid is used, although the solubility of glycogen is least, a further complication is introduced as the potassium chloride formed is only slightly soluble in alcohol, and large quantities of salt are precipitated along with the glycogen. This did not interfere with the acid hydrolysis, or with the estimation of sugar by the Hagedorn and Jensen method, but might with other methods. Since trichloroacetic acid is a definite protein precipitant, and its potassium salt is soluble in alcohol, it was decided to use this acid. Table IV shows a comparison of the result of neutralising with each of the three acids in an estimation of glycogen in muscle. 


\section{Table IV.}

After boiling frog muscle with potassium hydroxide the solution was diluted, three equal parts taken and neutralised with hydrochloric, glacial acetic and trichloroacetic acids respectively, the precipitates (of protein) were removed by centrifuging and the glycogen in an aliquot part of each solution precipitated with two volumes of alcohol and estimated.

\begin{tabular}{|c|c|c|c|c|c|}
\hline \multirow[b]{2}{*}{$\begin{array}{l}\text { Final vol. } \\
\text { of solution }\end{array}$} & \multicolumn{3}{|c|}{$\begin{array}{l}\text { mg. glycogen found when alkaline digest } \\
\text { is neutralised with }\end{array}$} & & \multirow{4}{*}{$\begin{array}{c}c-a \\
0.364\end{array}$} \\
\hline & $\begin{array}{c}\text { Glacial } \\
\text { acetic }\end{array}$ & $\begin{array}{l}\text { Hydro- } \\
\text { chloric }\end{array}$ & $\begin{array}{l}\text { Trichloro- } \\
\text { acetic }\end{array}$ & \multirow{3}{*}{$\begin{array}{l}b-a \\
0.416\end{array}$} & \\
\hline cc. & $(a)$ & (b) & $(c)$ & & \\
\hline 6 & 0.867 & $1 \cdot 283$ & $1 \cdot 231$ & & \\
\hline 6 & 0.495 & $0 \cdot 640$ & 0.629 & $0 \cdot 145$ & $0 \cdot 134$ \\
\hline 6 & 0.808 & 0.938 & 0.996 & 0.130 & 0.188 \\
\hline 6 & 0.496 & 0.472 & 0.976 & -0.024 & 0.480 \\
\hline 9 & $9 \cdot 06$ & $8 \cdot 87$ & - & -0.19 & - \\
\hline 9 & $3 \cdot 80$ & $4 \cdot 21$ & 一 & 0.41 & - \\
\hline 9 & $4 \cdot 74$ & - & $4 \cdot 92$ & - & 0.18 \\
\hline & $8 \cdot 71$ & & $8 \cdot 75$ & & 0.04 \\
\hline
\end{tabular}

The final procedure adopted was as follows. The solution was boiled with $60 \%$ potassium hydroxide for $2 \frac{1}{2}$ hours, cooled, neutralised with $40 \%$ tri-, chloroacetic acid, the final adjustment being made with dilute potassium hydroxide, so that the solution was faintly violet to bromocresol purple, the solution was made up to a known volume, centrifuged, and two volumes of alcohol were added to an aliquot part. Bromocresol purple was chosen as an indicator since it changes colour almost at neutrality, and since it is possible to see the colour even when working on muscle, which gives a brown solution. The glycogen precipitate was separated by centrifuging, washed once with absolute alcohol, hydrolysed with $2 \cdot 2 \%$ hydrochloric acid, and the sugar estimated either by the original Hagedorn and Jensen method [1923], or by Hanes's [1929] modification for use with larger quantities. It is advisable to wash the glycogen with absolute alcohol, since even $80 \%$ dissolves an appreciable fraction. A correction can be added for the glycogen lost by solution in the alcohol when precipitating, it can be found by reference to the potassium trichloroacetic acid curve (Fig. 2, curve $d$ ). Table $\mathrm{V}$ shows the result of estimating a solution of purified glycogen, $(a)$ by acid hydrolysis, $(b)$ by the method described above. The recovery of glycogen added to an estimation on frog muscle is also shown. The glycogen solution was added to a portion of the alkaline digest before neutralisation. In each case the recovery is low: this may be due to some substance present in muscle, which affects the solubility of glycogen, as noted above.

\section{Table V.}

mg. glycogen found in 2 cc. solution.

\begin{tabular}{|c|c|c|}
\hline $\begin{array}{l}\text { Estimated by acid } \\
\text { hydrolysis }\end{array}$ & $\begin{array}{l}\text { Estimated as described } \\
\text { in text }\end{array}$ & $\begin{array}{c}\text { Added in an estimation } \\
\text { on muscle }\end{array}$ \\
\hline $\left.\begin{array}{l}1 \cdot 36 \\
1 \cdot 34\end{array}\right\}$ & $\left.\begin{array}{l}1 \cdot 30 \\
1 \cdot 40\end{array}\right\}$ & $\left.\begin{array}{l}1 \cdot 31 \\
1 \cdot 28\end{array}\right\}$ \\
\hline $\left.\begin{array}{l}2 \cdot 12 \\
2 \cdot 14 \\
2 \cdot 17 \\
2 \cdot 14\end{array}\right\}$ & $\left.\begin{array}{l}2 \cdot 14 \\
2 \cdot 12 \\
2 \cdot 05 \\
2 \cdot 04\end{array}\right\}$ & $\left.\begin{array}{c}2.02 \\
2.26 \\
-\end{array}\right\}$ \\
\hline
\end{tabular}




\section{Solubility of glycogen in water.}

First the solubility of mussel glycogen, prepared as described, i.e. without boiling with alkali, was compared, at $20^{\circ}$, with that of a similar sample boiled for $2 \frac{1}{2}$ hours with $60 \%$ potassium hydroxide. The results are shown in Fig. 3 (curves $a$ and $b$ ). When the experiment was repeated, using a second preparation of glycogen from the same batch of mussel powder, the curves produced by plotting the results were not identical with those of the first preparation, but of a similar type (Fig. 3, curves $c$ and $d$ ). Glycogen prepared from a second batch of mussel powder, prepared similarly to the first, except that the mussels were killed and dehydrated by cold alcohol instead of boiling alcohol, gave a curve intermediate between those of the first two preparations (Fig. 3, curve $e$ ). In each case the solubility of glycogen which had been boiled with alkali had reached a practically stable value at the first estimation, whereas the glycogen not boiled with alkali required from 3 to 4 days for maximum solubility to be reached. In the first experiment after 5 days at $20^{\circ}$ the solutions were heated to $50^{\circ}$, and cooled again. The solubility of both samples of glycogen returned to the same value.

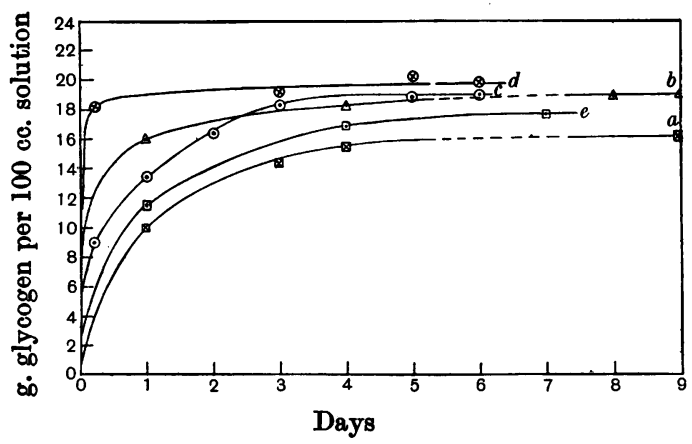

Fig. 3. Solubility of glycogen in water at $20^{\circ}$.

Prep. I. I( $a$ ) Not boiled with KOH $0.92 \mathrm{mg}$. nitrogen per $100 \mathrm{mg}$. glycogen.

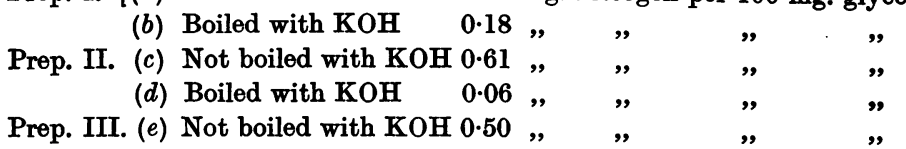

The solubility of mussel glycogen was then determined at $0^{\circ}$ and $37^{\circ}$ (Table VI). At $37^{\circ}$ all the glycogen $(0.4$ g.) had dissolved in the water added (1 cc.) forming a jelly. A portion of this jelly contained $40 \%$ glycogen, so probably the glycogen was evenly distributed and is soluble at least to this extent at $37^{\circ}$.

Table VI.

\begin{tabular}{|c|c|c|}
\hline \multicolumn{3}{|c|}{ Solubility of glycogen in water after 7 days at } \\
\hline $0^{\circ}$ & $20^{\circ}$ & $37^{\circ}$ \\
\hline $16 \%$ & $17 \cdot 7 \%$ & $>40 \%$ \\
\hline
\end{tabular}


The solubility of glycogen from mussels, frog muscle and rabbit liver was compared at $20^{\circ}$ (Fig. 4). The sample from rabbit liver had a higher solubility than either of the samples from cold blooded animals.

It seems possible that the increasing solubility of the samples not boiled with potassium hydroxide, reaching a value that approximates, but does not quite reach, that of the samples boiled with alkali, might be due to a depolymerising influence of water. If this is so, the boiling with alkali exerts the same effect at a much greater rate, and possibly to a greater extent. Schmid, Ludwig and Pietsch [1928] observed a somewhat similar effect when determining the molecular weight of glycogen in liquid ammonia, as their glycogen did not reach a steady state for half an hour, starting from a very large molecular weight and reaching a value of 180 . Their samples had presumably all been boiled with potassium hydroxide, since two were prepared commercially and no special mention is made of the preparation of the third sample (from liver). Possibly if estimations of the solubility of the glycogen boiled with potassium hydroxide had been made during the first

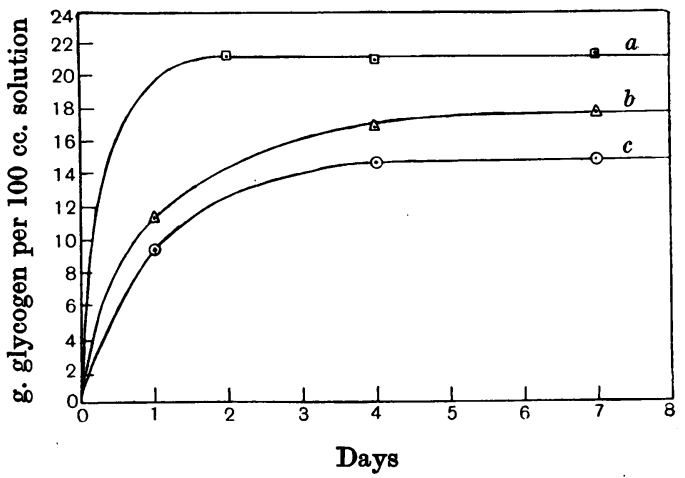

Fig. 4. Solubility of glycogen in water at $20^{\circ}$.

(a) Glycogen from rabbit; $0.12 \mathrm{mg}$. nitrogen per $100 \mathrm{mg}$. glycogen.

(b) " " mussels; $0.50 \mathrm{mg}$. " " "

(c) " " frog; $1.13 \mathrm{mg}$. " . " "

half hour a rising value would have been obtained, but there would have been no certainty that this was not due to the normally slow solution of a colloid. This does not necessarily suggest that glycogen in its natural state is polymerised to a large extent, since the glycogen prepared without boiling with alkali had been treated with alcohol in order to purify it, and it might well be this treatment which caused a high degree of polymerisation, if such existed. It is also possible that the nitrogen content of the glycogen is connected with the differences in solubility, as in most cases the rate of solution and the solubility vary inversely with the nitrogen content. 


\section{Summary.}

1. Glycogen from mussels, prepared without boiling with alkali, has been found to take from 3 to 4 days to reach saturation in water, whereas a similar sample boiled for $2 \frac{1}{2}$ hours with $60 \%$ potassium hydroxide, reaches a slightly higher value within a few hours.

2. The solubility in water of mussel glycogen, not boiled with potassium hydroxide, at $0^{\circ}$ is $16 \%$, at $20^{\circ} 17 \cdot 7 \%$ and at $37^{\circ}$ at least $40 \%$.

3. Glycogen from skeletal muscle of frogs has a solubility in water of $14.9 \%$ at $20^{\circ}$, that from rabbit liver $21 \%$ at $20^{\circ}$.

4. Glycogen is slightly soluble in aqueous alcohol, the concentration and nature of any electrolyte present having an influence on the value. Curves have been obtained showing the decreasing solubility of glycogen with increasing concentration of alcohol in the presence of potassium acetate, potassium trichloroacetate, potassium chloride, potassium hydroxide and trichloroacetic acid.

5. A micro-modification of Pflüger's method of estimating glycogen, based on the results of the experiments on the solubility of glycogen in aqueous alcohol, and employing a correction for this solubility, is described.

6. When glycogen prepared from mussels is precipitated with alcohol, after two precipitations the nitrogen content of the preparation increases, the phosphorus content remaining nearly constant.

I wish to express my thanks to Professor J. C. Drummond for his interest and advice in this work. The cost of the research has in part been defrayed by a grant from the Government Grant Committee of the Royal Society.

\section{REFERENCES.}

Bierry and Gouzon (1928). Compt. Rend. Soc. Biol. 99, 186.

Evans (1925). Biochem. J. 19, 1115.

- (1926). Physiol. Rev. 6, 367.

Hagedorn and Jensen (1923). Biochem. Z. 135, 46.

Hanes (1929). Biochem. J. 23, 99.

Holmes and Holmes (1926). Biochem. J. 20, 1196.

de Jongh and Planelles (1924). Biochem. Z. 154, 167.

Kahn (1922). Pflüger's Arch. 196, 400.

Pflüger (1904). Pflüger's Arch. 103, 169.

Slater (1924). Biochem. J. 18, 621.

Schmid, Ludwig and Pietsch (1928). Monatsh. Chem. 49, 118. 